\title{
A HOLOMORPHIC FUNCTION WITH WILD BOUNDARY BEHAVIOR
}

\author{
JOSIP GLOBEVNIK ${ }^{1}$
}

To the memory of Darja

\begin{abstract}
Let $B$ be the open unit ball in $\mathbf{C}^{N}, N>1$. It is known that if $f$ is a function holomorphic in $B$, then there are $x \in \partial B$ and an arc $\Lambda$ in $B \cup\{x\}$, with $x$ as one endpoint along which $f$ is constant. We prove

THEOREM. There exist an $r>0$ and a function $f$ holomorphic in $B$ with the property that, if $x \in \partial B$ and $\Lambda$ is a path with $x$ as one endpoint, such that $\Lambda-\{x\}$ is contained in the open ball of radius $r$ which is contained in $B$ and tangent to $\partial B$ at $x$, then $\lim _{z \in 1 .: \rightarrow x} f(z)$ does not exist.
\end{abstract}

We denote by $B$ the open unit ball in $\mathbf{C}^{N}, N>1$. For each $x \in \partial B$ and $r$, $0<r \leqslant 1$, let $D(x, r)$ be the open ball of radius $r$, contained in $B$ and tangent to $\partial B$ at $x$. We prove the following

THEOREM. There exist an $r>0$ and a function $f$ holomorphic in $B$ such that if $x \in \partial B$ and $\Lambda$ is a path contained in $D(x, r)$, except for its endpoint $x$, then $\lim _{z \in \Lambda, z \rightarrow x} f(z)$ does not exist.

It is known that $r$ in the Theorem has to be strictly smaller than 1 [2]; whether or not it can be arbitrarily close to 1 is an open question.

For each $x \in \partial B$ and each $\rho, 0<\rho<1$, let $H(\rho x)$ be the real hyperplane through $\rho x$, tangent to $\rho B$ at $\rho x$. If $R>0$ let

$$
W(\rho x, R)=\{y \in H(\rho x):|y-\rho x|<R\} .
$$

Thus $W(\rho x, R)$ is the relatively open ball in $H(\rho x)$ of radius $R$ centered at $\rho x$.

LEMMA 1. There is an $r>0$ with the following property: let $0<a<1$; there exists $L \in \mathbf{N}$, numbers $\rho_{l}, 1 \leqslant l \leqslant L, a<\rho_{1}<\cdots<\rho_{L}<\rho_{L+1}=1$, and numbers $R_{l}>0$, $1 \leqslant l \leqslant L$, such that $\overline{W\left(x, R_{l}\right)} \subset \rho_{l+1} B$ for every $x \in \partial\left(\rho_{l} B\right), 1 \leqslant l \leqslant L$, and for each $l, 1 \leqslant l \leqslant L$, there is a finite set $T_{l} \in \partial\left(\rho_{l} B\right)$ such that

(i) $\overline{W\left(x, R_{l}\right)} \cap H(y)=\varnothing$ whenever $x, y \in T_{l}, x \neq y, 1 \leqslant l \leqslant L$;

(ii) given any $y \in \partial B$ there exist $l, 1 \leqslant l \leqslant L$, and $z \in T_{l}$ such that if $\Lambda$ is a path joining a point in $a B$ with $y$, such that $\Lambda-\{y\} \subset D(y, r)$, then $\Lambda$ meets $W\left(z, R_{l}\right)$.

Received by the editors October 11, 1983.

1980 Mathematics Subject Classification. Primary 32A40.

${ }^{1}$ This work was supported by the Republic of Slovenia Science Foundation.

1985 American Mathematical Society $0002-9939 / 85 \$ 1.00+\$ .25$ per page 
Lemma 2. Let $0<a<1$. Let $L, \rho_{l}, R_{l}$, and $T_{l}, 1 \leqslant l \leqslant L$, be as in Lemma 1. Given $\varepsilon>0$ and $C<\infty$ there is a polynomial $P$ such that

(i) $\operatorname{Re} P>C$ on $\bigcup_{l=1}^{L} \bigcup_{x \in T_{l}} W\left(x, R_{l}\right)$;

(ii) $|P|<\varepsilon$ on $a B$.

Proof. Choose $\rho_{i}^{\prime}: a<\rho_{1}^{\prime}<\rho_{1}<\rho_{2}^{\prime}<\cdots<\rho_{L}^{\prime}<\rho_{L}<\rho_{L+1}^{\prime}<1$ such that for each $l, 1 \leqslant l \leqslant L, \overline{W\left(x, R_{l}\right)} \subset \rho_{l+1}^{\prime} B\left(x \in \partial\left(\rho_{l} B\right)\right)$. Fix $l, 1 \leqslant l \leqslant L$, and denote $W_{l}=\cup_{x \in T_{l}} \overline{W\left(x, R_{l}\right)}$. If $\delta_{l}>0$ and $C_{l}<\infty$, then, by Lemma 1(i), one can prove, similarly to the proof of Theorem 4 in [1], that there is a polynomial $P_{l}$ such that $\left|P_{l}\right|<\delta_{l}$ on $\rho_{l}^{\prime} B$ and $\operatorname{Re} P_{l}>C_{l}$ on $W_{l}$. If we choose $\delta_{l}$ and $C_{l}$ properly, then $P=\sum_{l=1}^{l} P_{l}$ will have all the required properties. This completes the proof.

Proof of The TheOREM. By Lemmas 1 and 2 there exist an $r>0$, a sequence $a_{n}$, $0<a_{1}<\cdots<1, \lim a_{n}=1$, and a sequence of sets $W_{n}, W_{n} \subset a_{n+1} B-a_{n} \bar{B}$, such that if $n \in \mathbf{N}, x \in \partial B$, and $\Lambda$ is a path joining a point in $a_{n} B$ with $x$, which satisfies $\Lambda-\{x\} \subset D(x, r)$, then $\Lambda$ meets $W_{n}$; moreover, for each $n \in \mathbf{N}, \delta_{n}>0$, and $C_{n}<\infty$ there is a polynomial $P_{n}$ such that $\left|P_{n}\right|<\delta_{n}$ on $a_{n} \bar{B}$ and $\operatorname{Re} P_{n}>C_{n}$ on $W_{n}$. If the sequence $C_{n}$ is chosen inductively to increase to $+\infty$ fast enough, and if the sequence $\delta_{n}$ is chosen to decrease to 0 fast enough, then the series $\sum_{n=1}^{\infty}(-1)^{n} P_{n}$ converges uniformly on compacta in $B$ to a function $f$ holomorphic in $B$ with the property: if $x \in \partial B$ and $\Lambda$ is a path with $x$ as one endpoint which satisfies $\Lambda-\{x\} \subset D(x, r)$, then

$$
\limsup _{z \in \Lambda, z \rightarrow x} \operatorname{Re} f(z)=+\infty, \quad \liminf _{z \in \Lambda, z \rightarrow x} \operatorname{Re} f(z)=-\infty .
$$

This completes the proof.

To prove Lemma 1, we first prove three lemmas.

Lemma 3. Let $x, y \in \partial B$ and $|x-y|>2 R / \rho$, where $0<\rho<1$ and $R>0$. Then $\overline{W(\rho x, R)} \cap H(\rho y)=\varnothing$.

Proof. Suppose $z \in \overline{W(\rho x, R)} \cap H(\rho y)$. Then $|z|^{2}<\rho^{2}+R^{2}$; i.e., $z$ $\in \overline{W(\rho y, R)}$ and, consequently, $\rho|x-y| \leqslant|z-\rho x|+|z-\rho y| \leqslant 2 R$, a contradiction.

Lemma 4. Let $0<r<1,0<\rho<1$, and $0<P<2^{1 / 2}$. Suppose $x, y \in \partial B$, and $|x-y|<P(1-\rho)^{1 / 2}$. Then $x$ and $y$ both lie on the same side of $H(\rho x)$. Moreover, $H(\rho x) \cap D(y, r) \subset W\left(\rho x, Q(1-\rho)^{1 / 2}\right)$, where $Q=(1-r) P+(2 r)^{1 / 2}$.

Proof. The first statement follows from the fact that $P<2^{1 / 2}$, which implies that $|x-y|<(2(1-\rho))^{1 / 2}$. Suppose $H(\rho x) \cap D(y, r)$ is not empty. Write $y=\alpha x+w$, $\rho<\alpha \leqslant 1,|w|^{2}+\alpha^{2}=1$. The center of $D(y, r)$ is at a distance of $|\rho-(1-r) \alpha|$ from $H(\rho x)$ and at a distance of $(1-r)\left(1-\alpha^{2}\right)^{1 / 2}$ from $\mathbf{R} x$. Consequently, $H(\rho x) \cap D(y, r) \subset W(\rho x, R)$, where

$$
\begin{aligned}
R=(1-r)\left(1-\alpha^{2}\right)^{1 / 2}+\left\{r^{2}-[\rho-(1-r) \alpha]^{2}\right\}^{1 / 2} \\
=(1-r)\left(1-\alpha^{2}\right)^{1 / 2}+\{[(1-\rho)-(1-r)(1-\alpha)] \\
\cdot[2 r-(1-\rho)+(1-r)(1-\alpha)]\}^{1 / 2} .
\end{aligned}
$$


Since $|x-y|<P(1-\rho)^{1 / 2}$, we have $(1-\alpha)^{2}+\left(1-\alpha^{2}\right)<P^{2}(1-\rho)$; hence $1-$ $\alpha<(1-\rho) P^{2} / 2$, and, consequently,

$$
R \leqslant(1-r)(1-\rho)^{1 / 2}\left(P / 2^{1 / 2}\right) \cdot 2^{1 / 2}+(2 r(1-\rho))^{1 / 2} .
$$

This completes the proof.

Lemma 5. Let $p \in \mathbf{N}$ and $x \in \partial B$. There exist a neighbourhood $U \subset \partial B$ of $x$, an $r_{0}>0$, and $M \in \mathbf{N}$ such that, for any $r, 0<r<r_{0}$, there are finite sets $S_{m} \subset U$, $1 \leqslant m \leqslant M$, such that $U \subset \bigcup_{m=1}^{M} \bigcup_{y \in S m}(y+r B)$ and $|y-z| \geqslant p r$ whenever $y$, $z \in S_{m}, y \neq z, 1 \leqslant m \leqslant M$.

Proof. Part 1. We prove the following. Let $W \subset \mathbf{R}^{2 N-1}$ be a bounded set and let $k \in \mathbf{N}$. There is a $\mu=\mu(k, N) \in \mathbf{N}$ such that, given any $r>0$, there are finite sets $T_{m} \subset \mathbf{R}^{2 N-1}, 1 \leqslant m \leqslant \mu$, such that $W \subset \bigcup_{m=1}^{\mu} \bigcup_{y \in T_{m}}(y+r B)$ (in this part $B$ is the open unit ball in $\mathbf{R}^{2 N-1}$ ) and $|y-z| \geqslant k r$ whenever $y, z \in T_{m}, y \neq z, 1 \leqslant m \leqslant \mu$.

To do this put $L=2 N-1$, choose $q \in \mathbf{N}$ such that $q>k L^{1 / 2}$, and put $\mu=q^{L}$. Let $r>0$. Define $S \subset \mathbf{R}^{L}$ by

$$
S=\left\{k r\left(s_{1}, s_{2}, \ldots, s_{L}\right): s_{i} \in \mathbf{Z}, 1 \leqslant i \leqslant L\right\} .
$$

Observe that $|y-z| \geqslant k r$ whenever $y, z \in S, y \neq z$. Further, let $P$ be the set of $\mu$ points in the cube $\left\{t \in \mathbf{R}^{L}: 0 \leqslant t_{i} \leqslant k r, 1 \leqslant i \leqslant L\right\}$, defined by

$$
P=\left\{(k r / q)\left(s_{1}, s_{2}, \ldots, s_{L}\right): s_{i} \in \mathbf{Z}, 1 \leqslant s_{i} \leqslant q, 1 \leqslant i \leqslant L\right\} .
$$

There are $\mu$ sets of the form $y+S$, where $y \in P$, and they have the following properties:

(a) if $y \in P$ and $z, w \in y+S, z \neq w$, then $|w-z| \geqslant k r$;

(b) $\mathbf{R}^{L}=\bigcup_{y \in P} \bigcup_{z \in y+S}(z+K)$,

where $K$ is the cube $\left\{t \in \mathbf{R}^{L}:\left|t_{i}\right|<k r / q, 1 \leqslant i \leqslant L\right\}$.

Since $q>k L^{1 / 2}$, it follows that $k r / q<r L^{-1 / 2}$; hence $K \subset r B$, which implies $\mathbf{R}^{L}=\bigcup_{y \in P} \bigcup_{z \in y+S}(z+r B)$. Now the assertion follows from the boundedness of $W$.

Part 2. There exist an open neighbourhood $U^{\prime} \subset \partial B$ of $x$, an open neighbourhood $V \subset \mathbf{R}^{2 N-1}$ of 0 , a constant $c>0$, and a map $\Psi$ from $V$ onto $U^{\prime}$ such that

$$
(1 / c)|u-v|<|\Psi(u)-\Psi(v)|<c|u-v| \quad(u, v \in V) .
$$

Let $U \subset U^{\prime}$ be a compact neighbourhood of $x$. The statement of the lemma now follows easily from Part 1 . This completes the proof.

Proof of Lemma 1. It is enough to prove the following. Let $x \in \partial B$. There exist $M \in \mathbf{N}, r>0$, a neighborhood $U \subset \partial B$ of $x$, and $a, 0<a<1$, such that the following holds: Given any $\rho_{1}, a<\rho_{1}<1$, there exist $R>0$ and $\rho_{m}, 1<m \leqslant M+$ $1, \rho_{1}<\rho_{2}<\cdots<\rho_{M}<\rho_{M+1}<1$, such that $\overline{W(y, r)} \subset \rho_{m+1} B\left(y \in \partial\left(\rho_{m} B\right)\right.$, $1 \leqslant m \leqslant M)$ and, for each $m, 1 \leqslant m \leqslant M$, there is a finite set $S_{M} \subset \partial\left(\rho_{m} B\right)$ such that

(i) $\overline{W(y, R)} \cap H(z)=\varnothing$ whenever $y, z \in S_{m}, y \neq z, 1 \leqslant m \leqslant M$;

(ii) given any $y \in U$ there exist $m, 1 \leqslant m \leqslant M$, and $z \in S_{m}$ such that if $\Lambda$ is a path joining a point in $\rho_{1} B$ with $y$, where $\Lambda-\{y\} \subset D(y, r)$, then $\Lambda$ meets $W(z, R)$. 
To prove this let $p=9$ and let $U, r_{0}$, and $M$ be as in Lemma 5. Choose $P$, $0<P<2^{1 / 2}$, and $r>0$ such that

$$
Q=(1-r) P+(2 r)^{1 / 2}<(2 M)^{-1 / 2}
$$

and

$$
8(1-r)+(2 r)^{1 / 2} / P<p
$$

Note that, by (2),

$$
8 Q / P<p
$$

Choose $a<1$ so close to 1 that

$$
\begin{gathered}
1 / 2<a, \\
1-a<r,
\end{gathered}
$$

and

$$
P((1-a) / 2)^{1 / 2}<r_{0} .
$$

Let $a<\rho_{1}<1$. Set $\vartheta=\left(1-\rho_{1}\right) /(2 M)$ and let

$$
\rho_{m}=\rho_{1}+(m-1) \vartheta \quad(1 \leqslant m \leqslant M+1) .
$$

Put $R=Q\left(1-\rho_{1}\right)^{1 / 2}$. By (1) and (4),

$$
\begin{aligned}
R & <(2 M)^{-1 / 2}\left(1-\rho_{1}\right)^{1 / 2}=\vartheta^{1 / 2}=\left(\rho_{m+1}-\rho_{m}\right)^{1 / 2} \\
& \leqslant\left(\rho_{m+1}^{2}-\rho_{m}^{2}\right)^{1 / 2} \quad(1 \leqslant m \leqslant M),
\end{aligned}
$$

so $\overline{W(y, R)} \subset \rho_{m+1} B\left(x \in \partial\left(\rho_{m} B\right), 1 \leqslant m \leqslant M\right)$.

Now let $\varepsilon=P\left(1-\rho_{1}\right)^{1 / 2} \cdot 2^{-1 / 2}$. By $(6), \varepsilon<r_{0}$. Furthermore, since $\left(1-\rho_{1}\right) / 2<$ $1-\rho_{m}(1 \leqslant m \leqslant M)$, it follows that $\varepsilon<P\left(1-\rho_{m}\right)^{1 / 2}(1 \leqslant m \leqslant M)$. By $(5), 1-\rho_{m}$ $<r(1 \leqslant m \leqslant M)$; since $P<2^{1 / 2}$, it follows, by Lemma 4, that if $y, z \in \partial B$, $|y-z|<\varepsilon$, then both $y$ and $z$ lie on the same side of $H\left(\rho_{m} y\right), 1 \leqslant m \leqslant M$, and furthermore,

if $1 \leqslant m \leqslant M$ and if $y, z \in \partial B,|y-z|<\varepsilon$, then every path $\Lambda$, which joins a point in $\rho_{m} B$ with $z$ and satisfies $\Lambda-\{z\} \subset$ $D(z, r)$, meets $W\left(\rho_{m} y, Q\left(1-\rho_{m}\right)^{1 / 2}\right) \subset W\left(\rho_{m} y, R\right)$.

Furthermore, since $\varepsilon<r_{0}$, it follows by Lemma 5 that there are finite sets $T_{m} \subset \partial B, 1 \leqslant m \leqslant M$, such that

(a) $|y-z| \geqslant p \varepsilon$ whenever $y, z \in T_{m}, y \neq z, 1 \leqslant m \leqslant M$;

(b) $U \subset \bigcup_{m=1}^{M} \bigcup_{y \in T_{m}}(y+\varepsilon B)$.

Define $S_{m}=\rho_{m} T_{m}(1 \leqslant m \leqslant M)$.

Suppose $1 \leqslant m \leqslant M$ and $y, z \in T_{m}, y \neq z$. By (a) and (3) it follows that

$$
\begin{aligned}
|y-z| & \geqslant p \varepsilon>8(Q / P) \varepsilon=8(Q / P) \cdot P\left(\left(1-\rho_{1}\right) / 2\right)^{1 / 2} \\
& =8 Q \cdot 2^{-1 / 2}\left(1-\rho_{1}\right)^{1 / 2}>4 R .
\end{aligned}
$$

By (4), $\rho_{m}>1 / 2$ so, by Lemma $3, \overline{W\left(\rho_{m} y, R\right)} \cap H\left(\rho_{m} z\right)=\varnothing$. This proves (i). Suppose $y \in U$. By (b) there exist $m, 1 \leqslant m \leqslant M$, and $z \in T_{m}$ such that $|y-z|<\varepsilon$. Consequently, (ii) follows from (7). This completes the proof. 


\section{REFERENCES}

1. J. Globevnik and E. L. Stout, Highly noncontinuable functions on convex domains, Bull. Sci. Math. 104 (1980), 417-434.

2. Holomorphic functions with highly noncontinuable boundary behavior, J. Analyse Math. 41 (1982), 211-216.

Institute of Mathematics, Physics and Mechanics, E.K. University of Ljubluana, Ljubljana, YugOSI.AVIA 